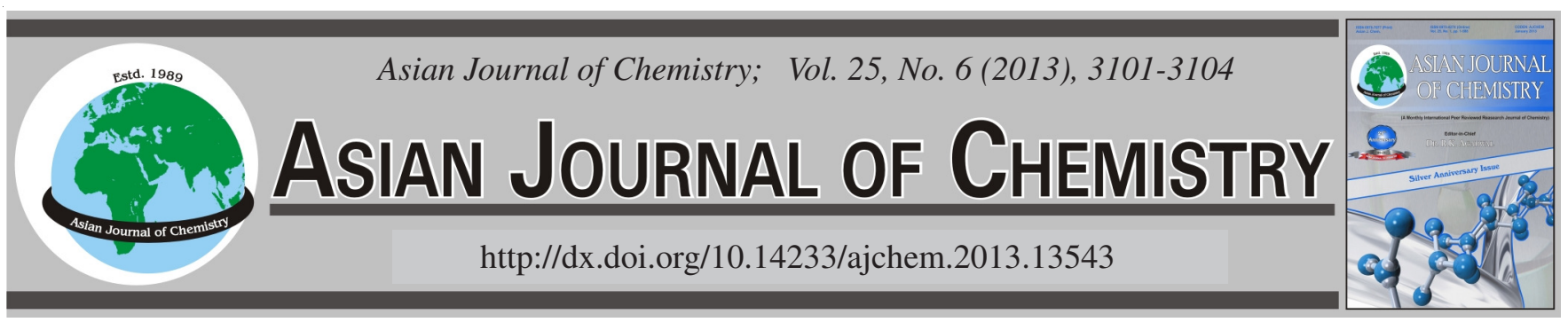

\title{
Growth of Cr-doped GaN Nanowires
}

\author{
Rongna Chen, Faming GaO*, Xiaohong GaO and Li Hou
}

Key Laboratory of Applied Chemistry, Yanshan University, Qinhuangdao 066004, P.R. China

*Corresponding author: Fax: +86 335 8061569; Tel: +86 335 8387552; E-mail: fmgao@ysu.edu.cn

Cr-Doped GaN nanowires were fabricated using a chemical vapour deposition method. The structures, morphologies and compositions of the products have been characterized by powder X-ray diffraction, transmission electron microscopy, selected area electron diffraction and energy dispersive X-ray analysis. Results revealed that the obtained nanowires are single-crystal GaN with hexagonal wurtzite structure. TEM images show two different morphologies, the bent and the very straight nanostructures. The optical property of Cr-doped $\mathrm{GaN}$ was observed in the photoluminescence spectra, which shows the as-prepared product emits a strong UV light emission at $395 \mathrm{~nm}$ and a yellow luminescence at $580 \mathrm{~nm}\left(\lambda_{\mathrm{ex}}=325 \mathrm{~nm}\right)$.

Key Words: Nanoparticles, Chemical vapour deposition, Crystal growth.

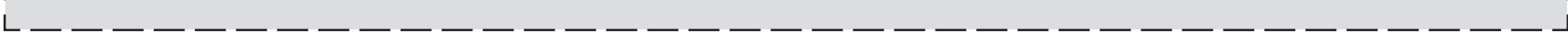

\section{INTRODUCTION}

Recently, there has been growing interest in the fabrication of GaN nanowires for their potential applications in optoelectronic and nanoscale electronic devices which take advantage of the unique high- aspect ratio structure of nanowires ${ }^{1-4}$. A variety of techniques have been utilized to synthesize GaN nanowires including chemical vapour deposition (CVD), hydride vapour-phase epitaxy, metal-organic chemical vapour deposition (MOCVD) and template-based growth method ${ }^{5-8}$. Among these method, chemical vapour deposition process in a horizontal furnace reactor is of particular interest due to the low cost of equipment and the simplicity of the experimental procedure. Moreover, GaN materials doped with transition metals, such as Mn, V, Ni and so on, have attracted much attention due to their peculiar optical electrical or magnetic properties ${ }^{9-14}$. In this letter, $\mathrm{Cr}$-doped GaN nanowires were successfully synthesized using catalyst-assisted chemical vapour deposition method by evaporating $\mathrm{Ga}_{2} \mathrm{O}_{3}$ powder under ammonia. The growth method allows a continuous synthesis and produces high purity $\mathrm{GaN}$ nanowires. The effect of $\mathrm{Cr}$-doping on the optical and structural properties of GaN nanowires was investigated.

\section{EXPERIMENTAL}

Preparation of GaN nanowires: A quartz boat holding both the substrate and $0.5 \mathrm{~g} \mathrm{Ga}_{2} \mathrm{O}_{3}$ powder $(99.99 \%)$ was placed inside a quartz tube in a horizontal tube furnace. Silicon substrates coated with $\mathrm{Ni}\left(\mathrm{NO}_{3}\right)_{2}$ were placed downstream of the $\mathrm{Ga}_{2} \mathrm{O}_{3}$ powder. The distance between the substrate and
$\mathrm{Ga}_{2} \mathrm{O}_{3}$ was $7 \mathrm{~mm}$. Then, the flowing $\mathrm{Ar}(99.999 \%$ ) gas was introduced into the tube to flush out the residual air for $5 \mathrm{~min}$ and then $\mathrm{NH}_{3}$ was flowed into the tube with a flow rate of $3 \mathrm{~L} / \mathrm{h}$ for $8 \mathrm{~h}$ at $900{ }^{\circ} \mathrm{C}$. After ammoniated, the flowing $\operatorname{Ar}(99.999 \%)$ gas was introduced into the tube to flush out the residual $\mathrm{NH}_{3}$ for $5 \mathrm{~min}$. After reaction, a light-yellow layer was found on the substrate surface.

Preparation of $\mathbf{C r}$-doped GaN nanowires: GaN nanowires were grown by chemical vapour deposition at $900{ }^{\circ} \mathrm{C}$. A $\mathrm{Ga}_{2} \mathrm{O}_{3}$ was used as the Ga source, $\mathrm{NH}_{3}$ as the $\mathrm{N}$ source and $\mathrm{CrCl}_{3} \cdot 6 \mathrm{H}_{2} \mathrm{O}$ as the $\mathrm{Cr}$ dopant source. $\mathrm{Si}(100)$ was used for substrate. $\mathrm{Ni}\left(\mathrm{NO}_{3}\right)_{2}$ was employed as catalyst, respectively. Prior to the growth of GaN nanowires, drying was performed in $\mathrm{Ar}$ ambient for $0.5 \mathrm{~h}$. Then, the growth temperature was adjusted to $900{ }^{\circ} \mathrm{C}$ in $\mathrm{NH}_{3}$ ambient and $\mathrm{GaN}$ nanowires were grown for $3 \mathrm{~h}$. After terminating the growth, the sample was cooled down in $\mathrm{NH}_{3}$ ambient from the growth temperature to $50^{\circ} \mathrm{C}$.

$\mathrm{X}$-Ray powder diffraction patterns were carried out on a $\mathrm{D} / \mathrm{max}-2500 / \mathrm{PC} \mathrm{X}$-ray diffractometer with $\mathrm{CuK}_{\alpha}$ radiation $(\lambda=$ $0.15418 \mathrm{~nm}$ ) to study the phases in presence. The morphologies of samples were characterized by transmission electron microscopy (TEM) using a JEM-2010 transmission electron microscope with EDS and a scanning electron microscope (SEM; XL30 ESEM FEG). Transmission electron diffraction (TED) was used to investigate the phase structure of the obtained powder. Room-temperature photoluminescence measurement of dry powder was recorded with a FL3-11 fluorescence spectrophotometer using an excitation wavelength of $325 \mathrm{~nm}$ and a slitwidth of $2 \mathrm{~nm}$. 


\section{RESULTS AND DISCUSSION}

Fig. 1 shows the XRD patterns of the undoped and $\mathrm{Cr}$ doped GaN nanowires grown on the silicon substrate. The five peaks (100), (002), (101), (110) and (103) of undoped GaN nanowires are located at $32.6,34.7,36.9,58.0$ and $63.7^{\circ}$, respectively, demonstrating that the reflections can be indexed to the hexagonal wurtzite $\mathrm{GaN}$ phase with the lattice constants $\mathrm{a}=0.318 \mathrm{~nm}$ and $\mathrm{c}=0.517 \mathrm{~nm}$. Compared with undoped $\mathrm{GaN}$, the d-space of (100) planes of the Cr-doped GaN increase from $0.274-0.280 \mathrm{~nm}$. Because the ionic radius of $\mathrm{Cr}$ and $\mathrm{Ga}$ are 0.69 and $0.62 \AA$, respectively, the doping of $\mathrm{Cr}$ induces the increase of the crystal constant of GaN nanowires. Both the spectra show that the peak (101) has the highest intensity. The intensity of the peak depends on the number of nanowires grown along the lattice orientation in the nanostructured films. The strongest (101) peak indicates that the maximum nanowires grow along [101] orientation. Moreover, no other peaks of impurities appears in the spectra, indicating the predominant single wurtzite GaN phase of the deposit and the sharp diffraction peaks also reveal that the GaN nanowires possess good crystalline quality. There is only one peak of the Si at $2 \theta=69.10^{\circ}$ with high intensity in this plot.

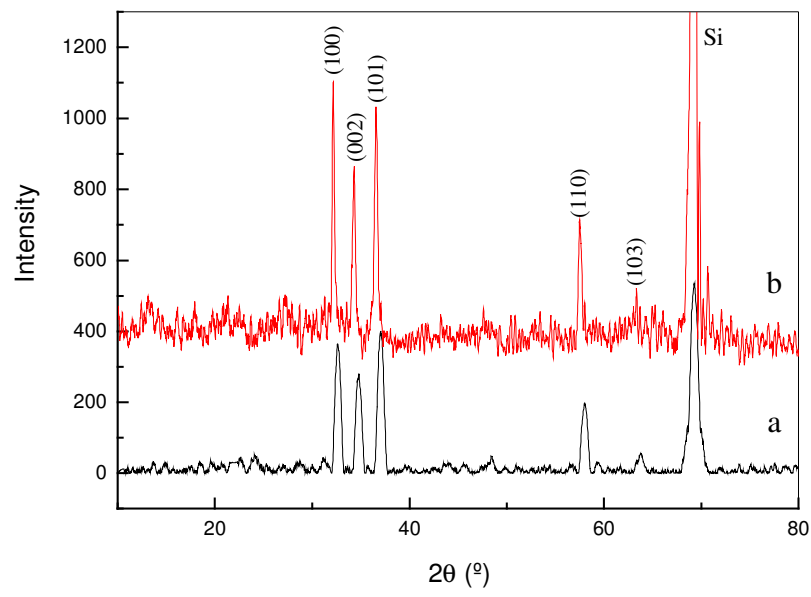

Fig. 1. X-Ray diffraction patterns of (a) undoped GaN and (b) Cr-doped GaN nanowires

The typical SEM images of the undoped GaN nanowires are shown in Fig. 2. Analysis of SEM micrographs indicates clearly that the sample contains a large number of wire-like nanostructures with typical lengths in the range of several tens to several hundreds of micrometers. These nanowires interwine with each other and homogeneously distributed over the whole surface of Si substrate. The range of the diameter of as-grown nanowires spans from $50-100 \mathrm{~nm}$ and small variation in diameter could been found along an individual nanowire as shown in Fig. 2(b).

Further observation reveals that there are solid particles located at the end of the nanowires. Energy dispersive X-ray spectrum (Fig. 2c) which was taken from the circle marked part in Fig. 2(b) shows that there are $\mathrm{Ga}, \mathrm{N}$ and $\mathrm{Ni}$ at the tip and the presence of small amount of Ni confirms that the solid particles are acturally metal nickel. It was concluded that the GaN nanowires were grown via the VLS mechanism by employing an $\mathrm{Ni}$ as a metal catalyst to initiate the seed of the

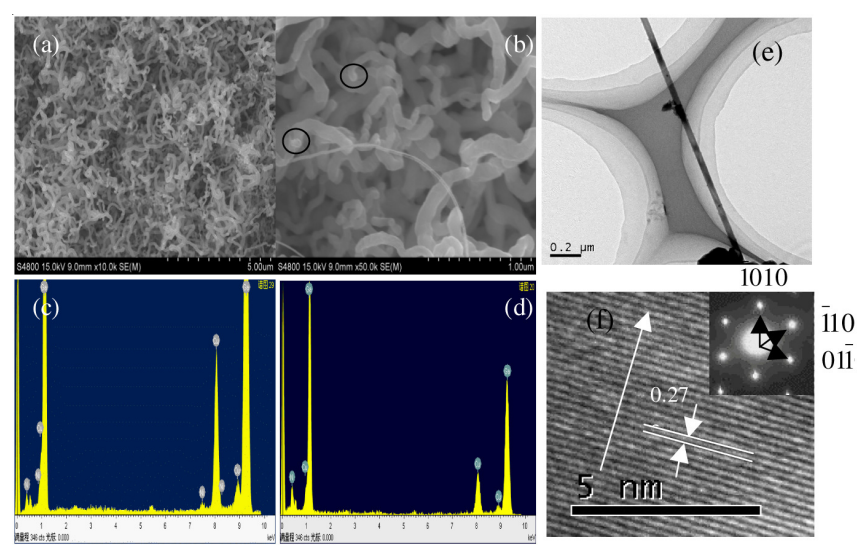

Fig. 2. FESEM images and EDX spectra of GaN nanowires. (a) Typical SEM image of GaN nanowires at low magnification; (b)Magnified image of GaN nanowires at local part; (c) The EDX spectrum taken from the top of GaN nanowires; (d ) the EDX spectrum taken from GaN nanowires; (e) The TEM image of a straight nanowires; (f) The corresponding HRTEM image of the nanowires. Inset: the SAED image of the GaN nanowires

GaN nanowires. Several EDS measurements taken from other parts of nanowire were used to estimate the stoichiometry of the wires. From the spectra as shown in Fig. 2(d), it can be seen that the nanowires are composed mainly of $\mathrm{N}$ atom and $\mathrm{Ga}$ atom, $(\mathrm{Cu}$ peaks are due to the copper grid used for TEM measurements). The $\mathrm{A} \%$ data of $\mathrm{N}$ atom and $\mathrm{Ga}$ atom is 49.42 and $50.58 \%$, which make stoichimetric GaN clearly. Fig. 2(e) shows a representative TEM image of a free-standing straight nanowire, which has diameter of $c a .53 \mathrm{~nm}$ and is $c a .60 \mu \mathrm{m}$ long. According to the observation of TEM, the estimated yield of the straight nanowires is less than $5 \%$. High-resolution TEM (HRTEM) was carried out to further understand the structural properties of GaN nanowires. Fig. 2(f) shows the HRTEM image of an individual $\mathrm{GaN}$ nanowire with a diameter of $c a .70 \mathrm{~nm}$. The well-spaced lattice fringe in the image indicate the single crystal structure of GaN nanowires with high crystalline quality but with less dislocations and defects. The measured spacing between adjacent lattice planes is 0.273 $\mathrm{nm}$, corresponding to (100) plane of as-prepared GaN, indicating that the nanowire preferably grew along the [100] direction. Further evidence for the formation of singlecrystalline nanowires could be found in selected area electron diffraction pattern. The insert in the upper-right-hand corner is the selected area electron diffraction pattern of the nanowire, which can be indexed to the reflection of single crystalline hexagonal wurtzite $\mathrm{GaN}$ with the growth direction parallel to the [100] direction of hexagonal unit cell.

The morphologies of Cr-doped GaN samples grown on the surface of $\mathrm{Si}$ substrate were investigated by TEM. The resulting sample shows two different morphologies, the bent and the very straight nanostructures. Fig. 3(a) is the typical low-magnification TEM image of the bent Cr-doped GaN nanowires. The nanowires were apt to grow with less bent structure, even very straight $\mathrm{Cr}$-doped $\mathrm{GaN}$ nanowires were synthesized with almost uniform diameter of $c a .50 \mathrm{~nm}$ as shown in Fig. 3(b). According to the observation of TEM, the estimated yield of the straight nanowires reaches to $c a .50 \%$. Fig. 3(c) show a representative TEM image of a free-standing nanowire, which is $c a$. $10 \mu \mathrm{m}$ long. 

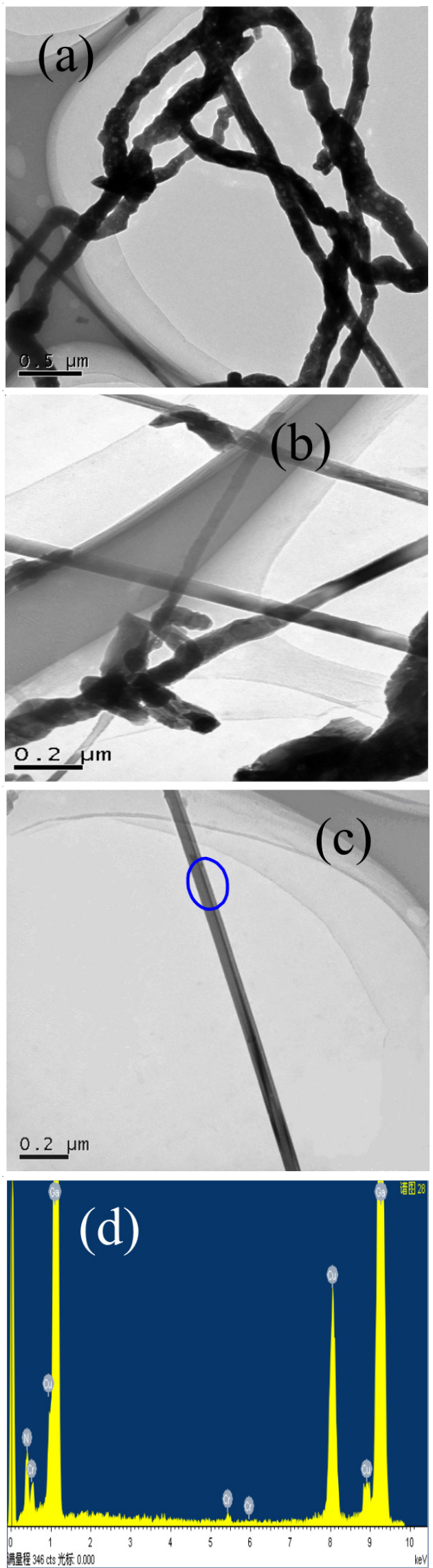

Fig. 3. TEM images of Cr-doped GaN nanowires. (a) TEM image of bent Cr-doped $\mathrm{GaN}$ nanowires; (b) TEM image of straight $\mathrm{Cr}$-doped $\mathrm{GaN}$ nanowires; (c) TEM image of an individual straight $\mathrm{Cr}$-doped $\mathrm{GaN}$ nanowires; (d) EDX spectrum of $\mathrm{Cr}$-doped $\mathrm{GaN}$ nanowire
The nanowire possess fairly straight morphology and also have a smooth surface without any particles. The representative EDS spectrum (Fig. 3d) taken from several parts of the straight nanowires shows that the nanowire is composed pre-dominantly of Ga-48 at. and N-49 at. \% and a small amount of $\mathrm{Cr}-3$ at. \%, suggesting the $\mathrm{GaN}$ nanowires are doped with Cr.

A further investigation on the microstructure of the Cr-doped GaN nanowires was performed by HRTEM. The HRTEM image as shown in Fig. 4a was taken from the the blue circle marked part in Fig. 3(c) and clearly shows the lattice fringes which are consistent throughout the crystal. It also reveals that the examined region is free from dislocation and stacking faults, whereas the surface of the wire terminated with thin (ca. $0.5 \mathrm{~nm}$ ) and smooth amorphous layers. Higher resolution TEM image as shown in Fig. 4b present the clear crystal lattice of nanowires. The crystal plane spacing is $c a$. $0.279 \mathrm{~nm}$, which is larger than that of (100) crystal plane spacing ( $0.274 \mathrm{~nm}$ ) of hexagonal GaN. Cr doping slightly changes the lattice constant of $\mathrm{GaN}$, which is in accordance with XRD result. The corresponding fast Fourier transform (FFT) pattern, shown in the inset of Fig. 4b, reveal that the nanowires is high quality single-crystal in nature. The spots on the pattern can be attributed to the [100] zone axis of hexagonal GaN. This indicates that the $\mathrm{Cr}$-doped $\mathrm{GaN}$ nanowires grew along the [100] direction.
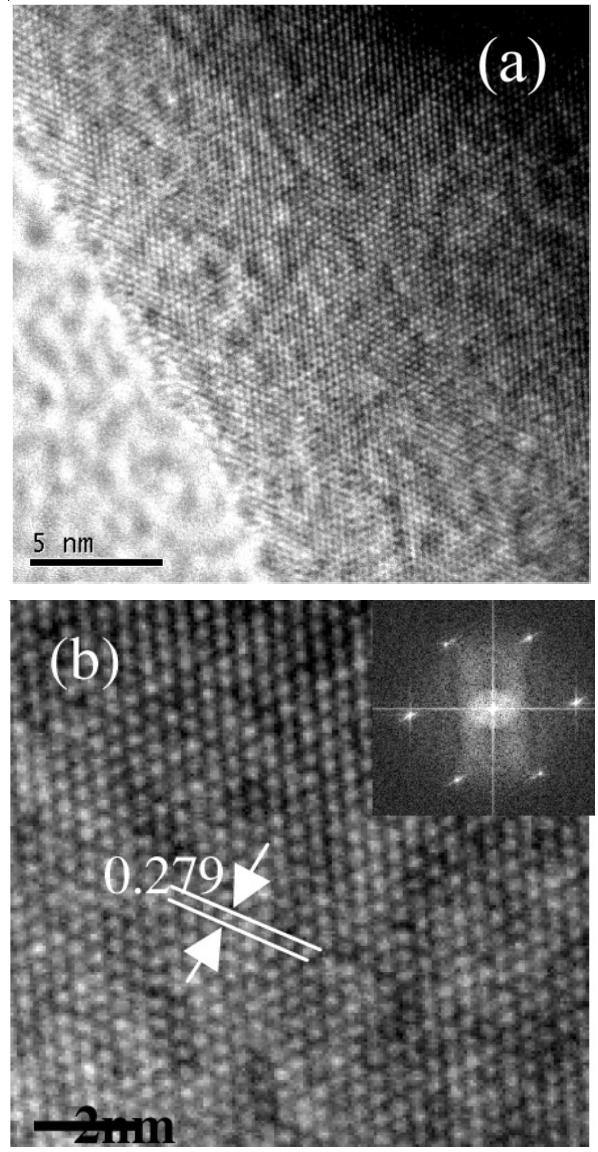

Fig. 4. HRTEM images of $\mathrm{Cr}$-doped GaN nanowires. (a) HRTEM image taken from the edge part of straight $\mathrm{Cr}$-doped $\mathrm{GaN}$ nanowires; (c) HRTEM image taken from the blue circle marked part in Fig. 4c. The inset shows the corresponding fast Fourier transform (FFT) image 
The optical properties of nanowires are important in understanding the quality of the materials and the potential application in optoelectronics. PL spectrum of GaN product presented in Fig. 5(a), which was detected with He-Cd laser as the excitation source (with a wavelength of $325 \mathrm{~nm}$ ) at room temperature $\left(22^{\circ} \mathrm{C}\right)$. A strong UV light emission peak at 388 $\mathrm{nm}$ is observed as shown in Fig. 5(a), which corresponds to the $\mathrm{GaN}$ near band-edge emisssion. Because GaN nanowires are too large for quantum confinement effects and the diameter of the thinnest nanowire is much larger than the Bohr exciton radius $(11 \mathrm{~nm}$ ) of $\mathrm{GaN}$, the PL emission has no blue shift but redshift from the bandgap emission compared with bulk $\mathrm{GaN}^{15}$. The optical properties of Cr-doped GaN nanowires are also shown in Fig. 5(b). Three emission peaks at 395, 532 and 580 $\mathrm{nm}$ are observed. The $395 \mathrm{~nm}$ emission should result from GaN band-edge emission and the small peak at $532 \mathrm{~nm}$ may attributed to deep-level emission ${ }^{16}$. The yellow emission at 580 $\mathrm{nm}$ might be ascribed to intrinsic point defects, such as Ga
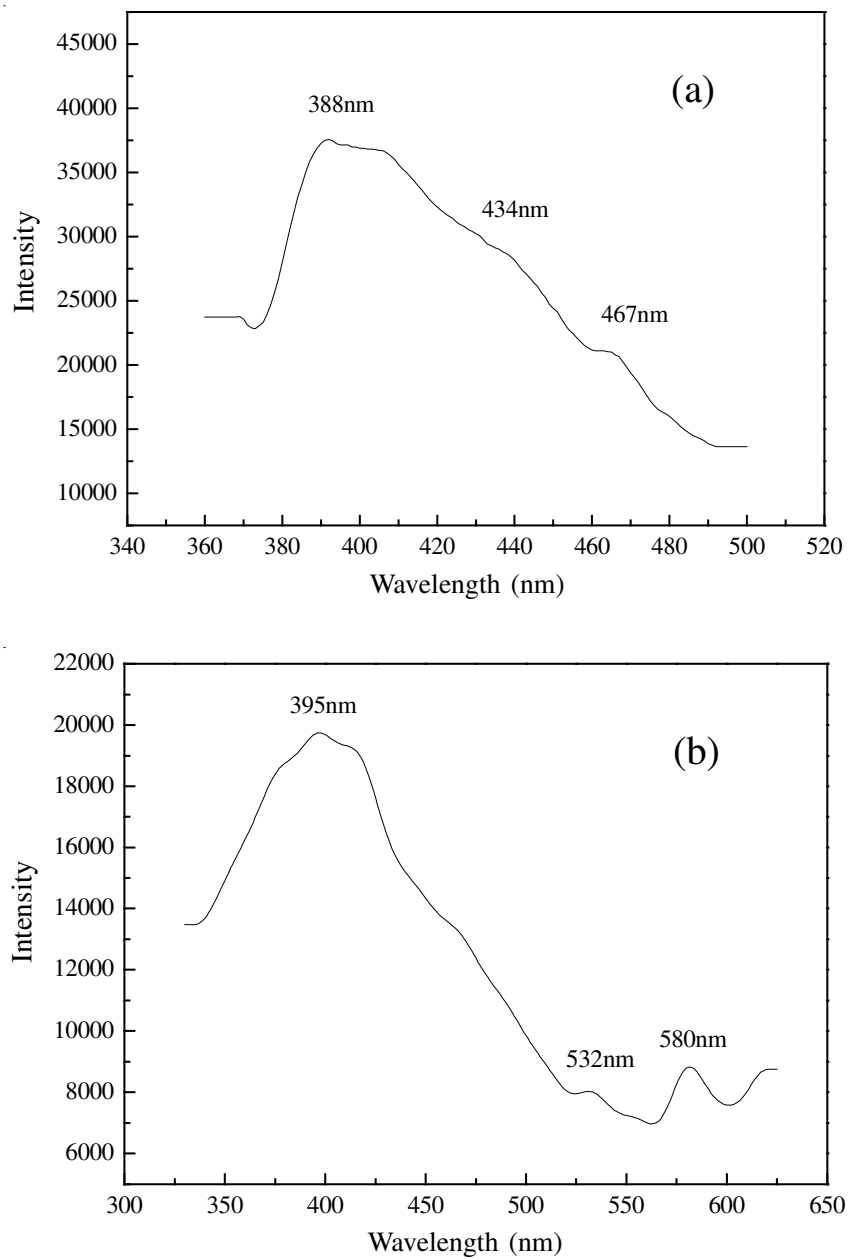

Fig. 5. Photoluminescence spectrum of the (a) undoped and (b) $\mathrm{Cr}$-doped $\mathrm{GaN}$ nanowire vacancies, to impurities or to complexes of intrinsic defects and impurities ${ }^{17}$. These defects would induce new energy levels in the band gap. Compared with the bulk GaN, both of the luminescence peaks of the undoped GaN and Gr-doped GaN nanowires produced a slight red shift. In the general consideration for photoluminescence spectra of one-dimensional structure, the red shift of the photoluminescence peaks is mainly caused by occasional defects such as point defects and stack faults. The as-synthesized GaN nanowires have good optical properties, which supply the advantage for the applications in photoelectron devices. However, further work is needed to investigate the photoluminescence mechanism of the GaN nanowires.

\section{Conclusion}

The Cr-doped GaN nanowires were successfully fabricated by chemical vapour deposition. The diameter of GaN nanowire was $c a .50 \mathrm{~nm}$ and their length was about several hundreds of micrometers. The PL measurement demonstrated that the undoped GaN nanowire has a stronge emission peak at $388 \mathrm{~nm}$. The typical emissions related to $\mathrm{Cr}$-doped $\mathrm{GaN}$ at $395 \mathrm{~nm}$ were also observed.

\section{ACKNOWLEDGEMENTS}

Financial support from the National Natural Science Foundation of China (Grant No. 21071122) and Research Fund for the Doctoral Program of Higher Education of China (Grant No. 20091333110009) and the Natural Science Foundation of Hebei (Grant No. E2010001169).

\section{REFERENCES}

1. L.S. Yu, Y.W. Ma and Z. Hu, J. Crystal Growth, 310, 5237 (2008).

2. $\quad$ X.T. Zhou, T.K. Shan and Y.Y. Shan, J. Appl. Phys., 97, 104315 (2005).

3. C.Y. Nam, D. Tham and J.E. Fischer, Appl. Phys. Lett., 85, 5676 (2004).

4. C.C. Chen, C.C. Yeh, C.H. Chen, M.Y. Yu, H.L. Liu, J.J. Wu, K.H. Chen, L.C. Chen, J.Y. Peng and Y.F. Chen, J. Am. Chem. Soc., 123, 2791 (2001).

5. X.M. Cai, A.B. Djurišic and M.H. Xie, Thin Solid Films, 515, 984 (2006).

6. X.Z. Wang, G.H. Yua and C.T. Lin, Solid State Commun., 150, 168 (2010).

7. B. Liu, R. Zhang and Z.L. Xie, J. Crystal Growth, 310, 4499 (2008).

8. L.G. Gai, H.H. Jiang and Y. Wan, J. Phys. Chem. C, 111, 2386 (2007).

9. Robert A. Burke and R. Daniel, J. Crystal Growth, 311, 3409 (2009).

10. F. Shi, D.D. Zhang and C.S. Xue, Mater. Sci. Eng. B, 167, 80 (2010).

11. M. Souissi, A. Bchetnia and B.E. Jani, J. Crystal Growth, 277, 57 (2005).

12. A.S. Otzlera and M. Deichera, Physica B, 340-342, 377 (2003).

13. G.P. Das, B.K. Rao and P. Jena, Phys. Rev. B, 68, 035207 (2003).

14. G.P. Das, B.K. Rao and P. Jena, Phys. Rev. B, 69, 214422 (2004).

15. B. 2004, Phys. Rev. B, 10, 676 (1974).

16. R.A. Burke, D.R. Lamborn, X.J. Weng and J.M. Redwing, J. Crystal Growth, 311, 3409 (2009).

17. R.Y. Korotkov, M.A. Reshchikov and B.W. Wessels, Physica B, 273274, 80 (1999). 